\title{
Mild ischemia produces hippocampal neuronal death in stroke-prone spontaneously hypertensive rats
}

Hidekazu Kinugawa, Yasuko Sakurai-Yamashita* and Masami Niwa

Department of Pharmacology1, Nagasaki University Graduate School of Biomedical Sciences, Nagasaki 852-8523, Japan.

* Address correspondence to Y. Sakurai-Yamashita, Department of Pharmacology1,

Nagasaki University Graduate School of Biomedical Sciences, 1-12-4 Sakamoto,

Nagasaki 852-8523, Japan

Tel: +81 95819 7043, Fax: +81958197044

E-mail: yasukosy@net.nagasaki-u.ac.jp

Number of text pages:20

Number of figures and tables: 4 figures and no tables 


\section{Abstract}

The blood flow in the hippocampus of stroke-prone spontaneously hypertensive rats (SHRSPs) and Wistar Kyoto (WKY) rats during occlusion of the carotid arteries was examined because it has been previously found that 2-vessel occlusion (2-VO) induces delayed neuronal death (DND) in the pyramidal cells of the CA1 hippocampal area in SHRSPs but not in WKY rats. DND was also examined in 4-week-old SHRSPs, which are as yet normotensive, in order to reveal the involvement of the development and maintenance of severe hypertension in DND in SHRSPs.

Before, during and after occlusion, the blood flow in the hippocampus was continuously monitored by laser Doppler flowmetry, wherein the probe was connected to a plastic fiber that was implanted in the CA1 subfield of animals. The change in blood flow was determined by comparing its rate during occlusion to the preoperative value. DND was confirmed by histological examination at 7 days after the operation.

The rate of blood flow during 2-VO was similar between the SHRSPs (42.6\% \pm $5.3 \%)$ and WKY rats $(49.0 \% \pm 14.3 \%)$. WKY rats that underwent 4 -vessel occlusion (4-VO), which induces DND in WKY rats, exhibited a severely decreased blood flow of $13.7 \%$ of the preoperative value. DND was also observed in 4-week-old SHRSPs that underwent 2-VO, and the incidence was identical to that in 12-week-old SHRSPs.

The present results suggest that the DND that occurs in SHRSPs due to 2-VO is not a result of the more severe reduction in blood supply during the occlusion than that in WKY rats, and secondary damage due to severe hypertension but is caused by some genetic factors due to which the pyramidal neurons of SHRSPs are more vulnerable to ischemic insult than those of WKY rats are.

\section{Classification: Section 8. Disease-Related Neuroscience}

Key words: ischemia, neuronal death, hippocampus, SHRSP, blood flow

Abbreviations: stroke-prone spontaneously hypertensive rats (SHRSPs), Wistar Kyoto (WKY), 2-vessel occlusion (2-VO), delayed neuronal death (DND), Monocyte chemoattractant protein-1 (MCP-1), hematoxylin-eosin (HE), glucose transporter-1 (GLUT-1) 


\section{Introduction}

Stroke-prone spontaneously hypertensive rats (SHRSP) develop severe hypertension and multisystem end-organ damage, and stroke occur spontaneously in these rats when they are kept on a high-salt diet, i.e., the SP diet with $0.8 \% \mathrm{NaCl}$ (Okamoto et al., 1974, Yamashita et al., 1990, Sakurai-Yamashita et al., 1997). In SHRSPs, delayed neuronal death (DND) of the pyramidal cells of the CA1 subfield of the hippocampus was observed after transient occlusion of the carotid arteries, i.e., 2-vessel occlusion (2-VO), which is observed in gerbils (Kirino et al., 1982, Yamashita et al., 1993, Yamashita et al., 1994, Qiang et al., 1989). Normally in rats, permanent occlusion of the vertebral arteries followed by transient occlusion of the carotid arteries, i.e., 4-vessel occlusion (4-VO), induces such DND (Pulsinelli et al., 1979). Wistar Kyoto (WKY) rats are used as controls because SHRSPs are generated from spontaneously hypertensive rats (SHRs) as stroke-prone strain, and SHRs are generated from WKY rats; (Okamoto et al., 1974, Yamori et al., 1974, Coyle et al., 1986, Coyle et al., 1986) this strain exhibits DND on 4-VO and not on 2-VO. The difference in the incidence of DND between SHRSPs and WKY rats may be a result of the difference in the blood supply in these rat strains, since it has been suggested that the blood flow in the cerebral collateral vessels is lower in hypertensive rats than in normotensive rats (Coyle et al., 1983, 1986). Furthermore, the severe hypertension in SHRSPs may damage the endothelial cells, or these rats could process other factors that influence the vulnerability of the hippocampal neurons to ischemic insult. The permeability of Evan's blue in the hippocampus of 16-week-old SHRSPs was higher than that in the hippocampus of WKY rats of the same age, suggesting that the activity of the blood-brain barrier (BBB) in the SHRSPs was weak; further, a prominent increase in dye permeability was noted in the SHRSPs immediately after 2-VO (Abraham et al., 2000). In SHRSPs, monocyte chemoattractant protein-1 (MCP-1), which increases the permeability of the BBB in vitro (Stamatovic et al., 2005), was strongly expressed in the astrocytes of the CA1 subfield of the hippocampus following 2-VO (Sakurai-Yamashita et al., 2006). The function of the BBB in SHRSPs might be altered.

The present study was designed to clarify whether the difference in blood supply during occlusion that results from alterations in the cerebral collateral vessels in SHRSPs affects the difference in the incidence of DND between SHRSPs and WKY rats. Furthermore, to study the involvement of severe hypertension in the incidence of DND, we conducted 2-VO in young SHRSPs of age 4 weeks which did not exhibit hypertension yet. No difference in blood supply during occlusion was observed between SHRSPs and WKY rats, and young SHRSPs also exhibited DND as adult SHRSPs at 
age of 12 weeks did.

\section{Results}

Fig 1-A and 1-B show the continuous blood flow in the CA1 subfield of the hippocampus of an SHRSP during the 2-VO operation. As observed in Fig 1-A, the blood flow decreased to $19 \%$ (indicated by an arrow) of the preoperative value (hereafter, prevalue) (arrowhead) when occlusion of the carotid arteries was initiated and it increased slightly within $1 \mathrm{~min}$; the average blood flow during occlusion was $36 \%$ of the prevalue. The blood flow immediately returned to normal after reperfusion. Fig 1-B shows another blood flow pattern during occlusion in the SHRSP. The blood flow clearly decreased to approximately $20 \%$ of the prevalue (indicated by an arrow) when the occlusion was started but within $3 \mathrm{~min}$, it increased to $>50 \%$ of the prevalue. The average blood flow during occlusion was $60 \%$ of the prevalue, and the blood flow immediately returned to normal after reperfusion. DND was confirmed in both animals at seven days after the operation (Fig 1-C and 1-D) irrespective of the rate of blood flow. Among the SHRSPs ( $n=5)$, the mean blood flow during the 2-VO was $42.6 \pm 5.3 \%$ of the prevalue.

Fig 2-A and 2-B show the blood flow during 2-VO in WKY rats. Fig 2-A is similar to Fig 1-A, which shows the blood flow in the SHRSP, and the average blood flow during the occlusion in the WKY rat was $24 \%$ of the prevalue. On the other hand, as indicated in Fig 2-B, the blood flow in another WKY rat almost returned to the prevalue at 2 min after occlusion was initiated. In this case, the average blood flow during the occlusion was $93 \%$ of the prevalue. DND induced by 2-VO was not observed in any WKY rat despite the decrease in blood flow during the operation to $<30 \%$ of the prevalue (Fig 2-C and 2-D). Among the WKY rats $(n=4)$, the mean blood flow during the $2-\mathrm{VO}$ was $49.0 \% \pm 14.3 \%$ of the prevalue.

In the case of the WKY rats, since DND was typically judged by $4-\mathrm{VO}$, the blood flow during the 4-VO operation was examined. Fig 3-A shows an example of the blood flow in WKY rats during the occlusion of the carotid arteries that was conducted after coagulation of the vertebral arteries. The blood flow decreased to less than $10 \%$ of the prevalue when the carotid arteries were occluded, and it remained low throughout the occlusion. The average blood flow during the $4-\mathrm{VO}$ was $12 \%$ of the prevalue. A severe decrease in the blood flow during occlusion and clear DND were observed in all the WKY rats that underwent 4-VO (Fig 3-B). Among the WKY rats $(n=3)$, the mean blood flow during 4 -VO was $13.7 \% \pm 4.4 \%$ of the prevalue.

Young 4-week-old SHRSPs which had not exhibited hypertension yet, were 
examined via the abovementioned experiments in order to clarify the influence of the development and maintenance of severe hypertension in SHRSPs on the occurrence of DND in the hippocampus. Of the 10 SHRSPs studied, 8 exhibited clear DND, as indicated in Fig 4. The rate at which DND occurred in 12-week-old SHRSPs was 74\% $(n=23)$, and no difference was observed in the incidence of DND between the 12- and 4-week-old of SHRSPs.

\section{Discussion}

The present study demonstrated that there was no difference in the blood flow during 2-VO between the SHRSPs and WKY rats. However the incidence of DND differed between these rats: the former exhibited DND, while the latter did not. These findings suggest that the extent to which the blood flow decreases during occlusion does not influence the occurrence of DND, and that even a mild reduction in blood flow induces DND in SHRSPs. Furthermore, since even young SHRSPs that did not exhibit hypertension exhibited DND, the secondary influence of severe hypertension is irrelevant to 2-VO-induced DND in SHRSPs.

SHRSP is an animal model of human cerebrovascular disease: as in the case of humans, these rats exhibit severe hypertension and spontaneously occurring strokes (Okamoto et al., 1974, Yamori et al., 1976, Saito et al., 1995, Sakurai-Yamashita et al., 1997, Qiang et al., 1989). It was found that in SHRSPs, DND was induced in the CA1 subfield of the hippocampus by transient occlusion of the carotid arteries, i.e., 2-VO (Yamashita et al., 1993, 1994, Qiang et al., 1989), although in normotensive rats, DND was induced by transient occlusion of the carotid arteries following the permanent occlusion of the vertebral arteries, i.e., 4-VO and not 2-VO (Pulsinelli et al., 1979). Since it has been suggested that the blood flow in the cerebral collateral vessels is lower in hypertensive rats than in normotensive rats (Coyle et al., 1986), the substantial decrease in the blood supply during 2-VO in the SHRSPs may be a reason underlying the high incidence of DND in these rats. However, the present study showed no relationship between the rate of blood flow during 2-VO and the incidence of DND in SHRSPs and WKY rats: in fact, the blood flow severely reduced to $12 \%$ of the prevalue during 4-VO in the WKY rats, and DND was induced. Furthermore, the severe hypertension in 12-week-old SHRSPs could damage endothelial cells and the BBB, thereby rendering neurons more susceptible to damage by ischemia and reperfusion. The blood pressure of the 4-week-old SHRSPs was around $120 \mathrm{mmHg}$, which is similar to that of normotensive rats. The high incidence of DND in such young SHRSPs strongly suggests that genetic factors other than hypertension could be responsible for 
the high vulnerability of hippocampal pyramidal neurons in SHRSPs to ischemic insults.

Several lines of evidence suggest differences in the function of the BBB between SHRSPs and WKY rats. The permeability of Evan's blue in the hippocampus was found to be higher in SHRSPs than in WKY rats at 16 weeks of age (Abraham et al., 2000). The abluminal to luminal ratio of the distribution of glucose transporter- 1 (GLUT-1) in SHRSPs was a quarter of that in WKY rats at 13 weeks of age, suggesting a difference in the polarity of the endothelial cells between these animals (Lippoldt et al., 2000). Although these data are provided by studies on severely hypertensive SHRSPs; unfortunately, no data is available with regard to younger animals that are as-yet normotensive. At age 20 weeks, SHRSPs exhibited lower expression of GLUT-1 in the endothelial cells and higher expression of aquaporin-4 in the astrocytes than WKY rats did: however, no such differences were observed in 6-week-old animals (Ishida et al., 2006). These results support the possibility that there could be differences in the BBB function between hypertensive SHRSPs and age-matched WKY rats. Nonetheless, they do not explain why the incidence of DND in young SHRSPs is high. Cultured cells derived from newborn SHRSPs indicated astrocytic dysfunction in these animals (Yamagata et al., 1997). Astrocytes are important components of the BBB that support endothelial cells (Abbott et al., 2006), and astrocytic degeneration induces microvascular damage and greatly increases the permeability of the BBB (Willis and et al., 2004). Therefore, in SHRSPs, astrocytes may not function adequately to support the functioning of the $\mathrm{BBB}$, and this may partly contribute to the high incidence of DND in these animals, because the permeability of the BBB in SHRSPs increased greatly due to 2-VO at 6 and $24 \mathrm{~h}$ after ischemia-reperfusion (Abraham et al., 2000). We found that in SHRSPs, MCP-1 was predominantly expressed in the astrocytes of the hippocampus from 8 to $48 \mathrm{~h}$ after 2-VO (Sakurai-Yamashita et al., 2006), and this protein may contribute to the increased BBB permeability (Stamatovic et al., 2005). Compared to WKY rat-derived astrocytes, SHRSP-derived astrocytes exhibited lower expression of the glial cell line-derived neurotrophic factor, GDNF on exposure to hypoxia/reoxygenation (Yamagata et al., 2002). The receptors for brain-derived neurotrophic factor were found to be mutated in SHRSPs (Kageyama et al., 1996). Since these neurotrophic factors could protect neurons from ischemic insults (Beck et al., 1994, Wang et al., 1997, Lin et al., 2006), this finding may be a reason underlying the difference in susceptibility to neuronal injury between SHRSPs and WKY rats. Genetic factors that determine astrocytic functions affect the BBB, and neurotrophic factors may be responsible for the occurrence of DND induced by 2-VO, a mild 
ischemic insult.

In summary, the present study demonstrated that DND in the hippocampus occurred due to mild ischemic insult, i.e., 2-VO, in both young and adult SHRSPs irrespective of whether or not the animals were hypertensive. This suggested that factors independent of cerebral collateral vessels and hypertension influence the occurrence of DND in SHRSPs. In the case of SHRSPs, genetic factors that have been inherited overgenerations, similar to those underlying the susceptibility to hypertension and stroke, could be responsible for the high vulnerability of the pyramidal cells of the CA1 hippocampal subfield to ischemic insult. Identification of these factors would provide therapeutic clues for the prevention of dementia in hypertensive patients.

\section{Experimental Procedures}

Animals: SHRSPs were bred and maintained at the Laboratory Animal Center for Biomedical Research, Nagasaki University School of Medicine. All the animals were provided access to the SP diet containing $0.8 \% \mathrm{NaCl}$ (Funabashi Farm Co., Chiba, Japan) and water ad libitum. Groups of 3 or 4 rats were housed in a cage in an air-conditioned room with a temperature of $24 \pm 1{ }^{\circ} \mathrm{C}$ and a humidity of $65 \% \pm 5 \%$ in a light:dark cycle of 12:12 h. All animals used for these procedures were treated in strict accordance with the NIH Guidelines for the Care and Use of Laboratory Animals and as approved by the Nagasaki University Animal Care Committee.

Surgical Procedures:

(1) Measurement of blood flow in the CA1 subfield of the hippocampus:

Male SHRSPs or WKY rats of age 11 weeks were injected with pentobarbital (60 $\mathrm{mg} / \mathrm{kg}$ ) and placed in the stereotaxic frame. A plastic fiber (length, $7 \mathrm{~mm}$; diameter, 0.5 $\mathrm{mm}$ ) was implanted into the pyramidal layer of the CA1 hippocampal subfield, $3.6 \mathrm{~mm}$ posterior and $2.1 \mathrm{~mm}$ lateral to the bregma, according to the stereotaxic co-ordinates of Paxinos and Watson (Paxinos et al., 1986). Seven days after the operation, the blood flow was continuously measured by laser Doppler flowmetry (Advance Laser Flowmetry, ALF21; Advance Co., Tokyo, Japan) wherein the probe was connected to the plastic fiber. The animals were anesthetized with $1.5 \%$ halothane in room air and their body temperature was maintained at $37^{\circ} \mathrm{C}$ with a heating pad. The left and right common carotid arteries were surgically exposed, and occluded for $10 \mathrm{~min}$ with aneurysmal clips (2-VO). For 4-VO (Pulsinelli et al., 1979), the right and left vertebral arteries of the male WKY rats in which the plastic fiber was implanted were irreversibly occluded by electrical coagulation, and the carotid arteries were exposed under pentobarbital (60 mg/kg, ip) anesthesia. On the following day, the animals were lightly 
anaesthetised with halothane, and the right and left common carotid arteries were occluded for 10 min with aneurysmal clips. The blood flow during the occlusion was determined as the average blood flow over 3 min just before occlusion was initiated. The rats were kept alive for 7 days after the measurement, after which they were sacrificed for examination of DND as well as for the histological assessment of the location of the fiber as described below.

(2) Histological evaluation of DND and the location of the fiber

Seven days after transient occlusion, the rats were deeply anesthetized and intracardially perfused with PBS followed by 4\% paraformaldehyde. Coronal paraffin sections (6 $\mu \mathrm{m}$ thick) at the level of plates 21-23 with the atlas as reference (Paxinos et al., 1986) were stained with hematoxylin-eosin (HE), and the loss of pyramidal cells in the CA1 hippocampal subfield was histologically evaluated. In all DND cases, this loss was observed. The location of the plastic fiber was also simultaneously examined by HE staining, and it was confirmed that it had been implanted in the CA1 subfield (indicated in supplement). The data obtained for animals in which the plastic fiber was not located in the CA1 hippocampal subfield were excluded.

\section{Acknowledgements}

This work was supported in part by grants from the Ministry of Education, Science and Culture, Japan. 


\section{Figure legends}

Fig 1 A chart showing the blood flow and a photograph of the pyramidal cells of the CA1 hippocampal subfield in SHRSPs subjected to 2-VO

(A) and (B) show the continuous blood flow during 2-VO. Note the immediate reduction in blood flow just after occlusion was initiated (indicated by an arrow). In the case of (A), the blood flow remained low, however, in the case of (B), the blood flow returned to $>50 \%$ the preoperative value within $3 \mathrm{~min}$. The vertical line represents the flowmetry values. The horizontal line represents the occlusion time.

(C) and (D) show the histochemical examination of pyramidal cells by HE staining. Clear DND is observed.

Fig 2 A chart showing the blood flow and a photograph of the pyramidal cells in the CA1 hippocmpal subfield in WKY rats subjected to 2-VO

(A) and (B) show the continuous blood flow during 2-VO. Note the immediate reduction in blood flow just after occlusion was initiated. In the case of (A), the blood flow remained low. However, in the case of (B), the blood flow was almost completely restored to the preoperative value. The vertical line represents the flowmetry values. The horizontal line represents the occlusion time.

(C) and (D) show the histochemical examination of pyramidal cells by HE staining. No DND is observed.

Fig 3 A chart showing the blood flow and a photograph of the pyramidal cells in the CA1 hippocampal subfield in WKY rats subjected to 4-VO

(A) shows the continuous blood flow during 4-VO. Note the immediate reduction in blood flow just after occlusion was initiated. It remained low throughout the occlusion. The vertical line represents the flowmetry values. The horizontal line represents the occlusion time.

(B) shows the histochemical examination of pyramidal cells by HE staining. Clear DND is observed.

Fig 4 Ratio of DND incidence in 12- and 4-week-old SHRSPs due to 2-VO

Male SHRSPs of age $12(n=23)$ and 4 weeks $(n=10)$ were operated by 2-VO. The 
ratio of DND was evaluated as the percentage of animals exhibiting DND. The open column indicates the ratio in the case of 12-week-old rats, and the black column indicates the ratio in the case of 4-week-old rats. 


\section{References}

Abbott, N.J., Ronnback, L., and Hansson, E. 2006. Astrocyte-endothelial interactions at the blood-brain barrier. Nat Rev Neurosci. 7,41-53.

Abraham, C.S., Harada, N., Deli, M.A., and Niwa, M. 2000. Transient forebrain ischemia increases the blood-brain barrier permeability for albumin in stroke-prone spontaneously hypertensive rats. Cell Mol Neurobiol. 22,455-462.

Beck, T., Lindholm, D., Castrén, E., and Wree, A. 1994. Brain-derived neurotrophic factor protects against ischemic cell damage in rat hippocampus. J Cereb Blood Flow Metab. 14, 689-692.

Coyle, P., and Heistad, D.D. 1983. Blood flow through cerebral collateral vessels in hypertensive and normotensive rats. Hypertension. 8 (II), 67-71.

Coyle, P., and Jokelainen, P.T. 1986. Differential outcome to middle cerebral artery occlusion in spontaneously hypertensive stroke-prone rats (SHRSP) and Wistar Kyoto (WKY) rats. Stroke. 14,605-611.

Ishida, H., Takemori, K., Dote, K. and Ito, H. 2006. Expression of glucose transporter-1 and aquaporin-4 in the cerebral cortex of stroke-prone spontaneously hypertensive rats in relation to the blood-brain barrier function. Am J Hypertens19(1):33-9. 19,33-39.

Jesmin, S., Togashi, H., Mowa, C.N, Ueno, K., Yamaguchi, T., Shibayama, A., Miyauchi, T., Sakuma, I. and Yoshioka, M. 2004. Characterization of regional cerebral blood flow and expression of angiogenic growth factors in the frontal cortex of juvenile male SHRSP and SHR. Brain Res. 1030,172-182.

Kageyama, H., Nemoto, K., Nemoto.F, Sekimoto, M., Nara, Y., Nabika, T., Iwayama, Y., Fukamachi, K., Tomita, I., Senba, E., Forehand, C.J., Hendley, E.D., and Ueyama, T. 1996. Mutation of the trkB gene encoding the high-affinity receptor for brain-derived neurotrophic factor in stroke-prone spontaneously hypertensive rats. Biochem Biophys Res Commun. 229,713-718.

Kirino, T. 1982. Delayed neuronal death in the gerbil hippocampus following ischemia. Brain Res. 239,57-69.

Lin, C.H., Cheng, F.C., Lu, Y.Z., Chu, L.F., Wang, C.H. and Hsueh, C.M. 2006. Protection of ischemic brain cells is dependent on astrocyte-derived growth factors and their receptors. Exp Neurol. 201,225-233.

Lippoldt, A., Kniesel, U., Liebner, S., Kalbacher, H., Kirsch,T., Wolburg, H. and Haller, H. 2000. Structural alterations of tight junctions are associated with loss of polarity in stroke-prone spontaneously hypertensive rat blood-brain barrier endothelial cells. Brain Res. 885,251-261.

Okamoto, K., Yamori, Y. and Nagaoka, A 1974. Establishment of the stroke-prone 
spontaneously hypertensive rat (SHRSP). Circ Res. 34,143-153.

Paxinos, G. and Watson, C. 1986. The Rat Brain in Stereotaxic Coordinates. Academic Press. New York.

Pulsinelli, W. A. and Brierley, J.B. 1979. A new model of bilateral hemispheric ischemia in the unanesthesized rat. Stroke. 10,267-272.

Qiang, W.G., Ikeda, K., Nara, Y., Horie, R. and Yamori, Y. $\quad$ 1989. Delayed neuronal death by transient ligations of bilateral carotid arteries in SHRSR, SHRSP and WKY. Jpn Heart J. 30,582.

Saito, H., Togashi, H., Yoshioka, M., Nakamura, N., Minami, M. and Parvez, H. 1995. Animal models of vascular dementia with emphasis on stroke-prone spontaneously hypertensive rats. Clin Exp Pharmacol Physiol Suppl. 22,S257-259.

Sakurai-Yamashita, Y., Shigematsu, K., Yamashita, K. and Niwa, M. 2006. Expression of MCP-1 in the hippocampus of SHRSP with ischemia-related delayed neuronal death. Cell Mol Neurobiol. 26,821-829.

Sakurai-Yamashita, Y., Yamashita, K., Kataoka, Y., Himeno, A., Niwa, M. and Taniyama, K. 1997. Expression of endothelin receptors and nitric oxide synthase in the brain of stroke-prone spontaneously hypertensive rats with cerebral apoplexy. Brain Res. 756,61-67.

Stamatovic, S.M., Shakui, P., Keep, R.F., Moore, B.B., Kunkel, S.L., Van Rooijen, N. and Andjelkovic, A.V. 2005. Monocyte chemoattractant protein-1 regulation of blood-brain barrier permeability. J Cereb Blood Flow Metab. 25,593-606.

Wang, Y., Lin S.Z., Chiou, A.L., Williams, L.R. and Hoffer, B.J. 1997. Glial cell line-derived neurotrophic factor protects against ischemia-induced injury in the cerebral cortex. J Neurosci. 17,4341-4348.

Willis, C.L., Nolan, C.C., Reith, S.N., Lister, T., Prior, M.J., Guerin, C.J., Mavroudis, G. and Ray, D.E. 2004. Focal astrocyte loss is followed by microvascular damage, with subsequent repair of the blood-brain barrier in the apparent absence of direct astrocytic contact. Glia. 45,325-337.

Yamagata, K., Tagami, M., Ikeda, K., Tsumagari, S., Yamori, Y. and Nara, Y. 2002. Differential regulation of glial cell line-derived neurotrophic factor (GDNF) mRNA expression during hypoxia and reoxygenation in astrocytes isolated from stroke-prone spontaneously hypertensive rats. Glia. 37,1-7.

Yamagata, K., Tagami, M., Nara, Y., Fujino, H., Kubota, A., Numano, F., Kato, T. and Yamori, Y. 1997. Faulty induction of blood-brain barrier functions by astrocytes isolated from stroke-prone spontaneously hypertensive rats. Clin Exp Pharmacol Physiol.24,686-691. 
Yamashita, K., Kataoka, Y., Niwa, M., Shigematsu, K., Himeno, A., Koizumi, S. and Taniyama, K. 1993. Increased production of endothellins in the hippocampus of stroke-prone spontaneously hypertensive rats following transient forebrain ischemia: histochemical evidence. Cell Mol Neurobiol. 13,15-23.

Yamashita, K., Niwa, M., Kataoka, Y., Shigematsu, K.,Himeno, A., Tsutsumi, K., Nakano-Nakashima, M.,Sakurai-Yamashita, Y., Shibata, S. and Taniyama, K. 1994. Microglia with an Endothelin ETB receptor aggregate in rat hippocampus CA1 subfields following transient forebrain ischemia. J Neurochem. 63,1042-1051.

Yamashita, K., Ohta, H., Uchida, S., Shigematsu, K., Ohkubo, T., Niwa, M. and Ozaki, M. 1990. Systemic effects of carteolol, a beta-adrenoceptor antagonist in stroke-prone spontaneously hypertensive rats. J Pharmacobiodyn. 13,64-69.

Yamori Y, Horie R, Handa H, Sato M and Fukase M 1976. Pathogenetic similarity of strokes in stroke-prone spontaneously hypertensive rats and humans. Stroke. 7,46-53.

Yamori, Y., Nagaoka, A. and Okamoto, K., 1974. Importance of genetic factors in hypertensive cerebrovascular lesions. Jpn Circ J. 38,1095-1100. 

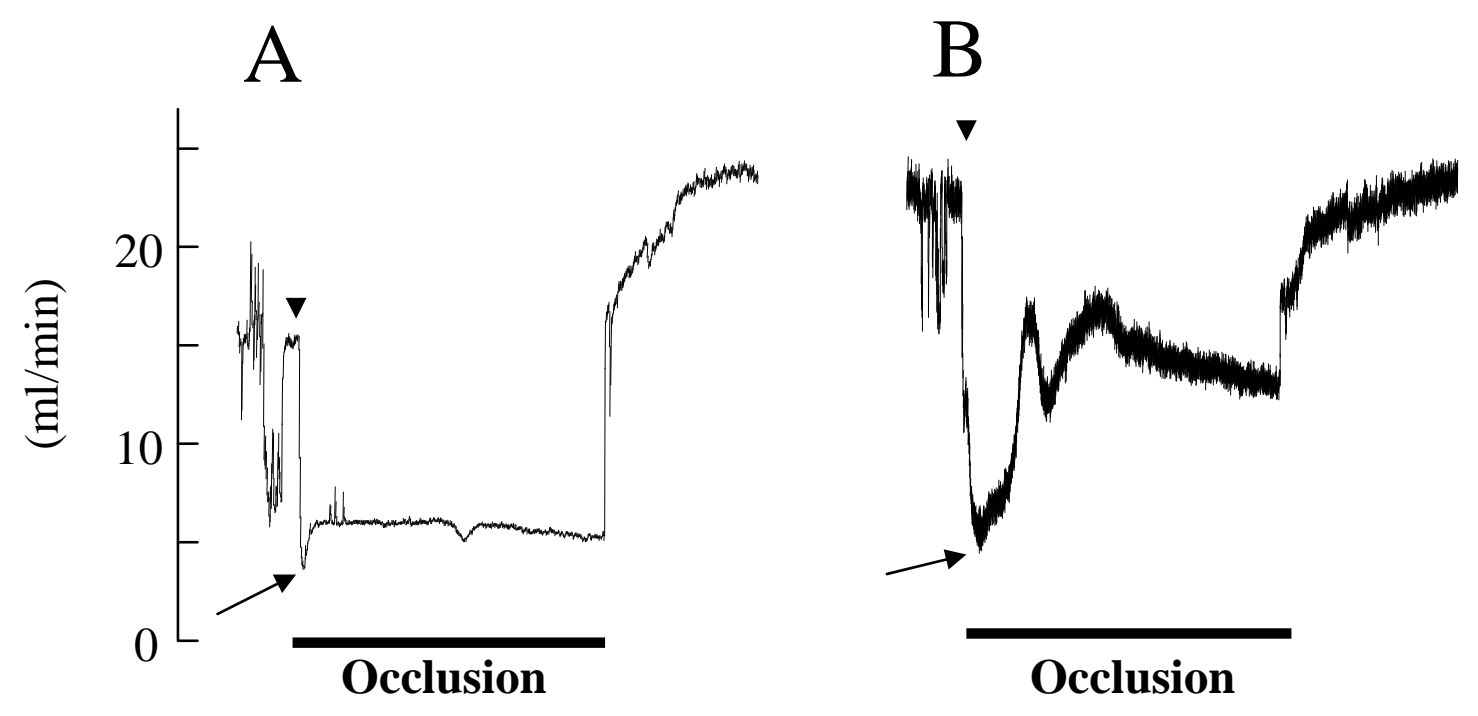

C
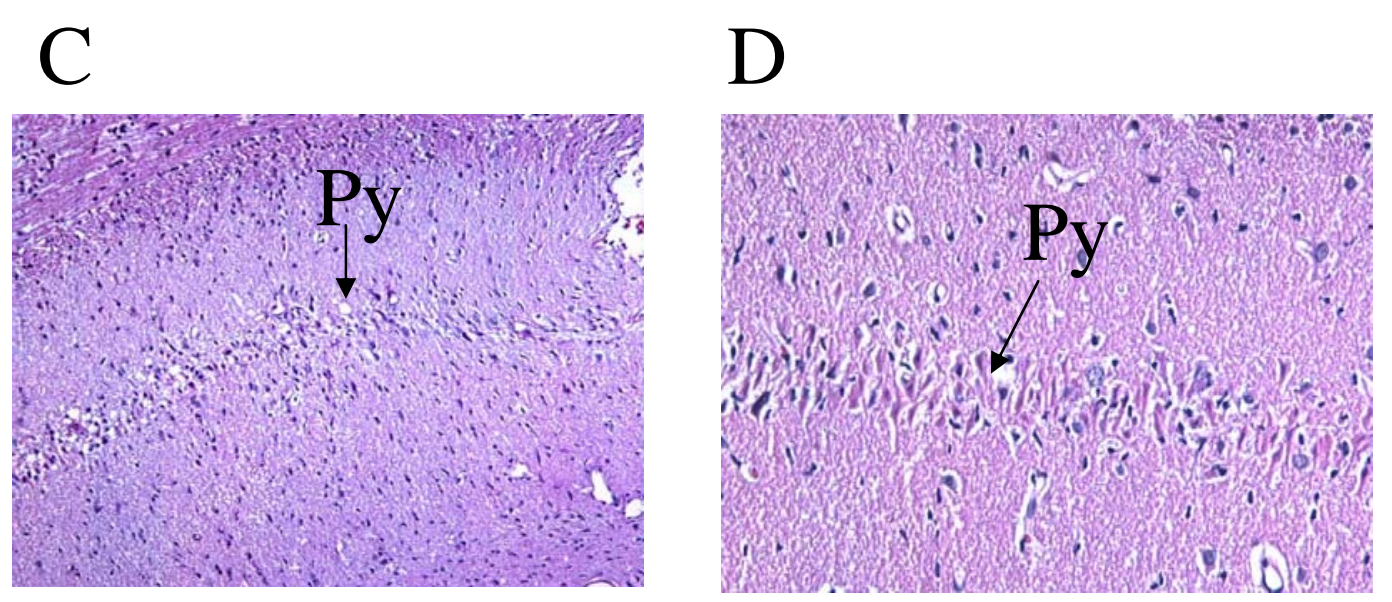

Fig 1 

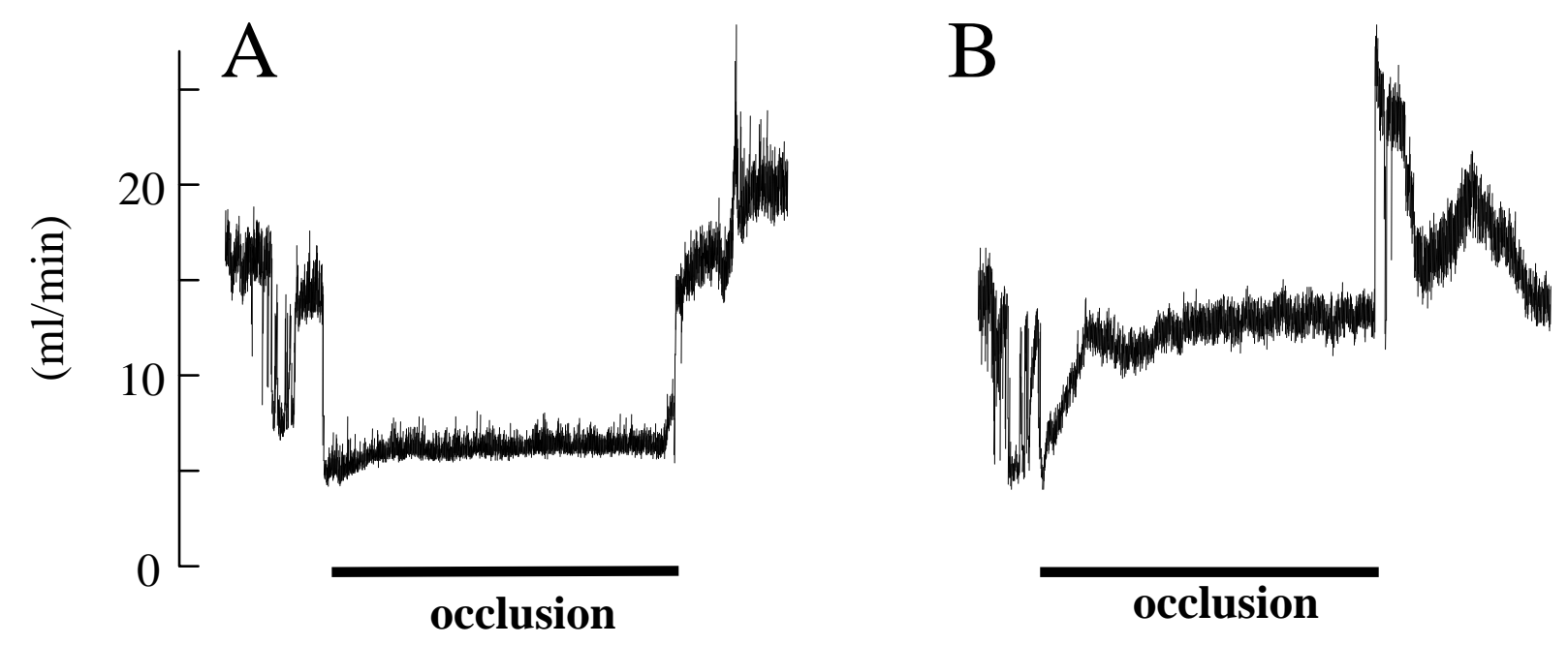

C
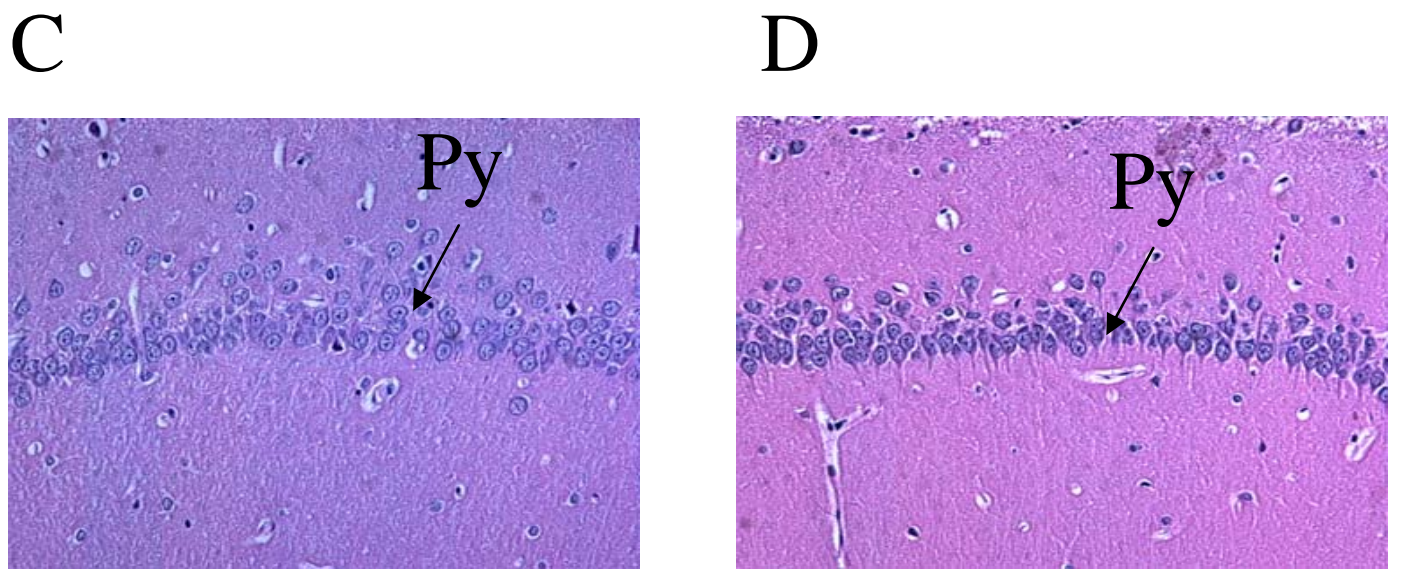

Fig 2 


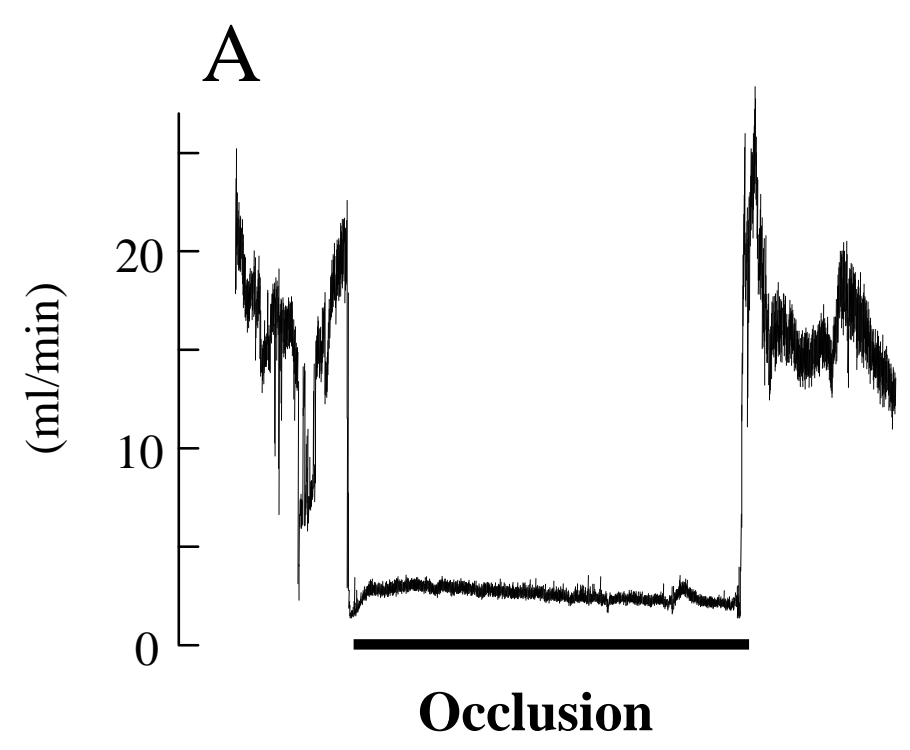

\section{B}

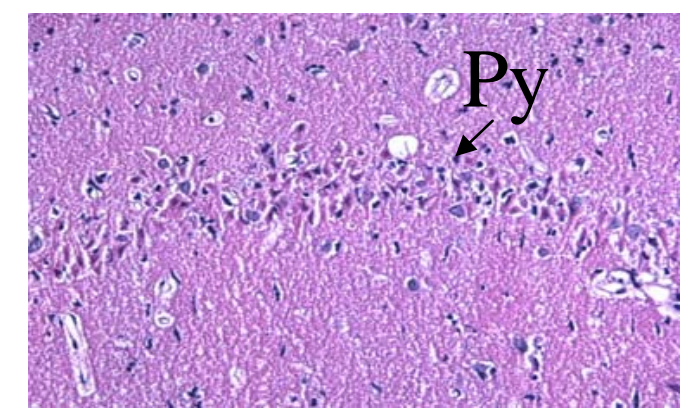

Fig 3 


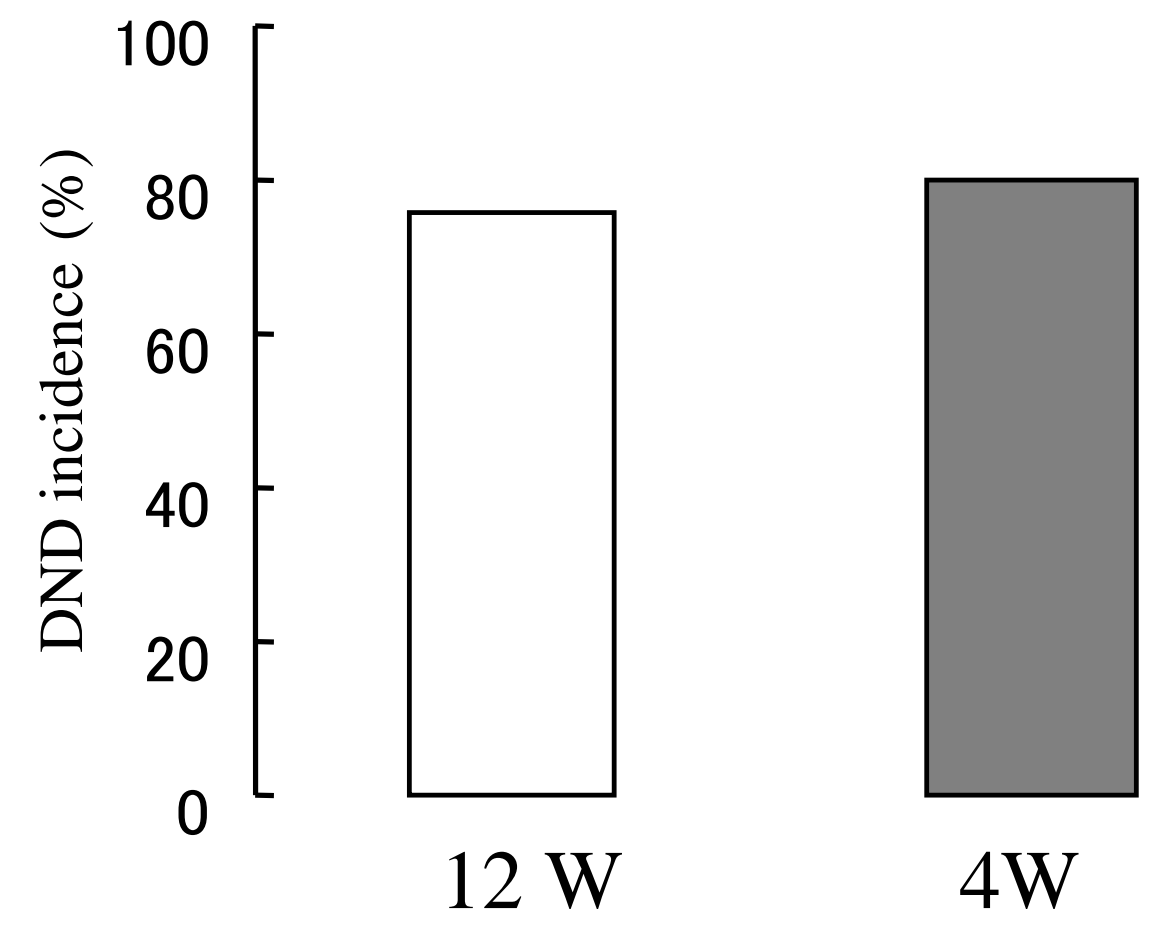

Fig 4 


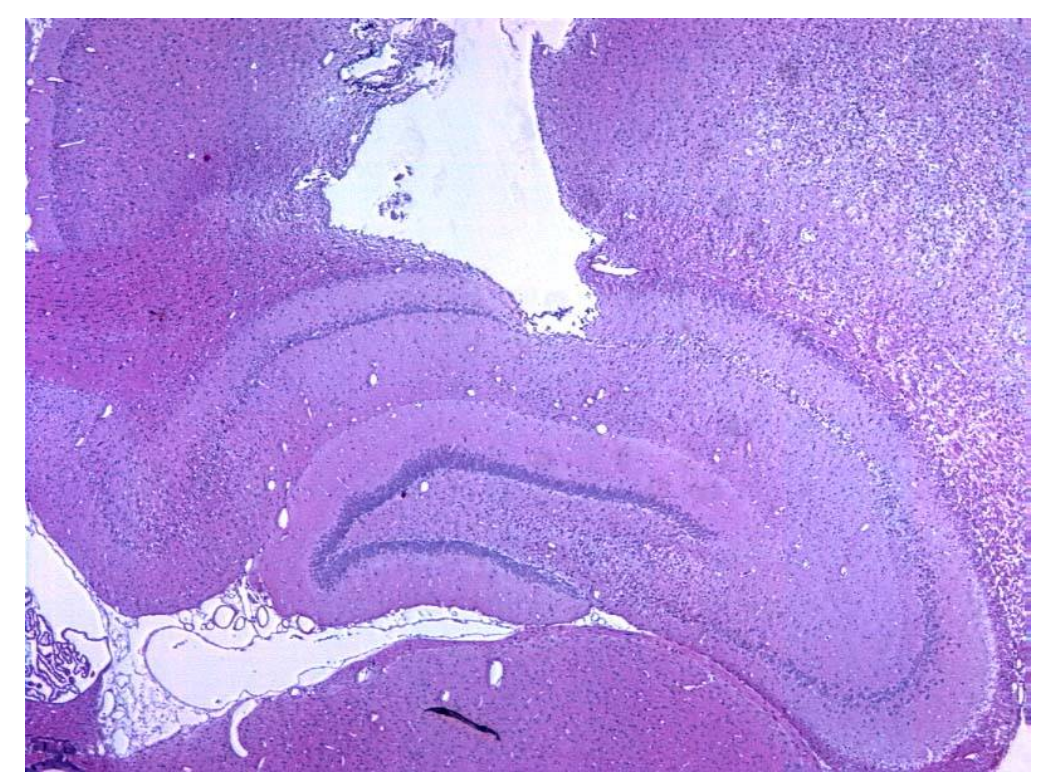

Supplement : The location of a plastic fiber in the CA1 hippocampal sufield

The tip of the plastic fiber was located in the CA1 hippocampal subfield. 